\title{
e-Migrinter
}

$18 \mid 2019$

L'ethnographie en migration(s)

\section{Réalisation d'un film ethnographique dans une ville périphérique israélienne}

Amandine Desille

\section{CpenEdition}

Journals

Édition électronique

URL : https://journals.openedition.org/e-migrinter/1670

DOI : 10.4000/e-migrinter. 1670

ISSN : 1961-9685

Éditeur

UMR 7301 - Migrinter

Référence électronique

Amandine Desille, "Réalisation d'un film ethnographique dans une ville périphérique israélienne », eMigrinter [En ligne], 18 | 2019, mis en ligne le 12 septembre 2019, consulté le 20 mai 2021. URL : http:// journals.openedition.org/e-migrinter/1670; DOI : https://doi.org/10.4000/e-migrinter.1670

Ce document a été généré automatiquement le 20 mai 2021.

Tous droits réservés 


\title{
Réalisation d'un film ethnographique dans une ville périphérique israélienne
}

\author{
Amandine Desille
}

\section{NOTE DE L'AUTEUR}

Les recherches menant aux présents résultats ont bénéficié d'un soutien financier du septième programme-cadre de l'Union européenne (7ePC/2007-2013) en vertu de la convention de subvention $n^{\circ}$ 316796. Je remercie Aurore Vermylen, Anne Bouhali et les évaluateurs anonymes pour leurs précieux commentaires.

\section{Introduction}

1 Il y a quarante ans, alors que le cinéaste Jean Rouch, créateur du "cinéma-vérité ", révolutionnait l'ethnographie visuelle (de Hasque, 2014, p. 41), et «inaugur[ait] une nouvelle conception du cinéma scientifique» (Gehin; Giglio-Jacquemont, 2013), faire un film ethnographique nécessitait de s'équiper, et de s'attendre à un peu de résistance à l'heure d'imposer la caméra. Le matériel était volumineux, et il était difficile pour l'ethnographe de se faire discret. Aujourd'hui cependant, il semble plus aisé de brandir un téléphone ou une petite caméra et de capturer une scène sans attirer l'attention, tant cette pratique est devenue commune et accessible à toutes et à tous. Ces évolutions technologiques (appareils photos ayant une fonction caméra, téléphones, caméras «embarquées »...) et leur démocratisation participent ainsi de la mobilisation grandissante de l'audiovisuel en sciences sociales (Gehin; Giglio-Jacquemont, 2013). Néanmoins, et alors même que le matériel se fait plus discret et la pratique plus ordinaire, les questionnements suivants ne cessent d'être réactualisés: quelle réciprocité instaurer avec les sujets filmés? Quelle est la part de l'interprétation de l'ethnographe? «Comment rendre visible sans stigmatiser?» (Raulin; et al., 2016). 
S'interroger sur sa place, alors que l'ethnographie filmique induit une posture « impliquée » (de Hasque, 2014), et sur la place de son équipement audiovisuel, reste au centre des préoccupations du chercheur qui inclut le film ethnographique dans ses méthodes d'enquête.

2 Pour répondre à ces préoccupations et encadrer au mieux la mobilisation de méthodes audiovisuelles, un nombre croissant de formations doctorales, d'écoles d'été et même

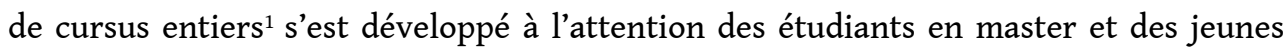
chercheurs en sciences sociales. Ces formations proposent de faire de la caméra l'outil principal de récolte puis de restitution des données, et articulent apprentissage technique (de l'usage de la caméra comme des logiciels d'édition) et réflexions autour des questions de réciprocité entre le chercheur et ses enquêtés, d'interprétation des données récoltées et d'éthique de la recherche. Bien que de nombreuses disciplines de sciences sociales ne permettent pas encore de présenter un film plutôt qu'un manuscrit à la fin de la thèse, de plus en plus de films ethnographiques sont produits par les chercheurs ${ }^{2}$.

3 Dans ce contexte, je me suis tournée vers l'ethnographie visuelle à l'occasion d'un appel à candidature ${ }^{3}$. J'ai alors proposé un projet de recherche, dans l'idée de filmer un court documentaire qui servirait de support à mes entretiens. L'objectif central du projet était de réaliser un film qui me permettrait d'engager un dialogue avec mes enquêtés autour des questions liées à la représentation politique des immigrés dans les localités qui constituaient alors mon terrain d'étude. Initialement, je souhaitais donc créer un outil méthodologique pour faciliter la collecte de données. Au fil du travail, le film a dépassé ce premier objectif pour devenir une méthode d'enquête à part entière, dont le résultat a été analysé au même niveau que les autres données récoltées au travers d'entretiens, d'observations participantes et d'analyses de la littérature grise.

Ce projet de film ethnographique est venu s'intégrer dans un travail de doctorat, qui examinait la production d'échelles locales de pouvoir à travers le cas spécifique de la politique d'accueil israélienne (Desille, 2017). Ce travail explore les politiques d'accueil de villes périphériques qui se caractérisent par une diversité des origines des habitants, liée aux vagues successives d'immigration. Dans ce contexte, ce projet de recherche souligne l'importance du rôle des acteurs - élus locaux et agents municipaux - dans la formulation et la mise en œuvre d'une politique d'accueil locale des immigrés.

Partant de ce postulat, j'ai imaginé un film centré sur un des acteurs de ma recherche, un homme politique local, immigré de Russie ${ }^{4}$, adjoint au maire d'une petite ville frontalière du nord de la Galilée, membre du parti nationaliste russophone Israel Beitenu $u^{5}$ et représentant de ce même parti pour le district. En suivant ce personnage pendant quelques jours, je souhaitais faire le portrait d'un homme politique immigré, et montrer les tensions qui pouvaient émerger du fait qu'il était à la fois un conseiller municipal, un membre d'un parti national, et un représentant communautaire. Le jour des élections nationales israéliennes en mars 2015, cet élu allait faire campagne au nom du parti Israel Beitenu. Mon idée consistait alors à le suivre ce jour-là caméra au poing. La réalisation d'un film m'a ainsi permis de justifier ma présence à ses côtés pendant cette journée. Aux questions qui me furent posées, je répondis que j'étais étudiante et que le film faisait partie de mon travail de doctorat. Cette filature aurait été difficile à expliquer autrement. J'ai ainsi pu observer et enregistrer les faits et gestes de cet élu local et ses interactions avec des résidents de la ville heure après heure. J'ai complété ces enregistrements quelques mois plus tard en tournant de nouvelles images lors des 
cérémonies commémoratives de la Seconde Guerre mondiale ${ }^{6}$. Dans la petite ville où mon personnage principal réside, anciens combattants et rescapés de la Shoah, décorés de médailles, se retrouvent en effet chaque $9 \mathrm{mai}^{7}$, date de ces commémorations, pour fleurir le monument aux morts, prendre quelques photos et déjeuner avec le maire.

6 En regardant les quatre heures d'enregistrement, accumulées lors de ces deux tournages, j'ai été frappée par la cohabitation, grinçante souvent, heureuse parfois, entre les résidents de la ville, fils et filles des immigrés juifs nord-africains des années 1950, et les «nouveaux» immigrés russophones arrivés dans les années 1990. Les blagues fusaient. Les résidents s'interpelaient en usant de l'origine nationale, utilisant des termes tels que le « Russe ", ou le " Marocain » pour se désigner. Ils s'accusaient les uns et les autres de ne pas "être d'ici », pour finalement s'accorder sur le fait que les juifs ne forment qu'un peuple, et qu'Israël est leur État. Devant cette confusion, ce chaos audio-visuel, j'ai sélectionné les interactions qui me paraissaient rendre compte au mieux de la complexité des acteurs, des actions, et de l'histoire, et j'ai monté un film intitulé Victory Day d'une durée de 17 minutes ${ }^{8}$.

7 Faisant l'hypothèse que le public serait majoritairement au fait de l'histoire israélienne, j'ai pris la décision de ne pas surcharger le film et n'ai pas précisé où se trouvait la ville de l'enquête, Kiryat Shmona, et quelle était sa "raison d'être " là, à quelques kilomètres du Liban et de la Syrie (Desille, 2018). Je me suis donc plutôt concentrée sur une série de rencontres, en débutant, grâce au montage, à l'inverse de la chronologie du tournage, par la cérémonie commémorative, qui atteste de la présence d'un groupe important d'immigrés d'ancienne Union soviétique, puis en me focalisant sur le bureau local du parti Israel Beitenu le jour des élections, en chroniquant les rencontres du représentant du bureau local avec les militants et avec les autres résidents de la ville, pour finalement revenir au bureau local assister à l'annonce télévisée des résultats. Dans ce film se superposent ainsi des images d'une ville périphérique à la frontière israélo-libanaise, d'un immigré russe en Israël devenu homme politique local, de sa communauté vieillissante, et de la cohabitation entre cette communauté russophone et les habitants "orientaux», juifs d'Afrique du Nord et du Moyen-Orient, établis là depuis les années 1950.

8 En m'appuyant sur cet exercice, je souhaite montrer dans quelle mesure la réalisation de ce film ethnographique a permis de soulever de nouveaux questionnements, autrement difficiles à faire émerger en s'appuyant sur des méthodes d'enquête plus classiques. En effet, il s'agit ici de mettre à jour l'intérêt méthodologique associé à la méthode filmique, dans une situation où le chercheur ne prend pas le film comme un miroir de la "réalité ", mais comme le résultat du croisement d'enjeux variés - ceux liés à la recherche, au tournage, à ce que les acteurs veulent ou peuvent montrer, et plus tard au montage. Pour ce faire, la première partie de l'article interrogera la production d'images en mouvement dans le contexte national israélien plus large. La deuxième partie décrira les nouveaux éléments, complémentaires des entretiens et des observations menés dans le cadre du doctorat, qui sont apparus lors du tournage. Le personnage principal du film, comme la majorité de mes interlocuteurs pendant le travail du terrain, est en charge d'élaborer la politique d'accueil de la ville. Comme dans d'autres villes où j'ai enquêté, cette dernière est souvent mise en œuvre par des membres du parti Israel Beitenu, situés à l'extrême droite de l'échiquier politique. Cette appartenance politique a une large influence sur l'encadrement et les termes de l'« intégration », mais aussi sur nos conversations. Dans ce contexte, la troisième partie 
montrera dans quelle mesure l'adoption d'une méthode visuelle permet de créer une distance avec les participants à la recherche, et de libérer la parole. Dans la quatrième partie, j'expliciterai les choix, fruits de la subjectivité du chercheur, qui ont mené au montage. Enfin, la dernière partie sera consacrée aux réactions des enquêtés euxmêmes, puis d'un public universitaire plus large, lors du visionnage du film finalisé. Cette dernière partie montrera les limites de ce premier film ethnographique.

L'adoption de la caméra a permis d'une part de dépasser les discours institutionnels pour mettre à jour les interactions sociales qui façonnent la ville et le vivre ensemble ; et d'autre part, de me positionner par rapport à des questions de racisme ordinaire, à Kiryat Shmona, mais aussi face à l'idéologie politique du parti Israel Beitenu et de ses représentants, parti auquel sont affiliés plusieurs participants à ma recherche.

\section{Filmer en Israël}

10 Alors que le conflit israélo-palestinien fait l'actualité internationale de façon hebdomadaire, et que de nombreuses images d'Israël parviennent chaque semaine au monde entier, la production d'images en mouvement dans une ville située à la frontière israélo-libanaise n'est pas un exercice anodin. Dans ce contexte de surproduction visuelle, la réalisation d'un film ethnographique répondait au désir de faire le portrait d'un lieu et des interactions qui s'y matérialisent, et ce afin de montrer ces ensembles d'interactions au lieu d'événements "spectaculaires ", de discours officiels ou même de récits romancés (Raulin ; et al., 2016 ; Weber, 2018). En ce sens, les méthodes d'enquête audio-visuelles ont généralement été considérées comme des méthodes privilégiées pour étudier des groupes moins "vocaux», comme les migrants ou les membres de minorités nationales par exemple, et permettre d'augmenter leur visibilité dans l'espace public, médiatique et aussi scientifique (Pereira; Maiztegui-Oñate; MataCodesal, 2016).

11 Mobiliser une méthode d'ethnographie filmique alors même que le terrain de l'enquête, de petites villes israéliennes situées aux frontières, est un espace marginal et peu connu, était un choix délibéré. De plus, Kiryat Shmona apparait rarement dans les médias internationaux lorsqu'il est question d'Israël. J'ai ainsi trouvé deux articles dans les archives de journaux français comme Le Monde ou Libération ${ }^{9}$, ou dans la presse américaine comme le New York Times ${ }^{10}$, lorsque le conflit israélo-libanais place la ville sur le front des tensions dans les années 1980, puis lors de la dernière guerre israélolibanaise en 2006. Kiryat Shmona apparaît également une fois dans ces quotidiens étrangers alors que l'équipe de football locale gagne un championnat en 2012, mais le titre surnomme l'équipe "Israeli underdog $»^{11}$, ce qui en dit long sur la position marginale de cette ville. Même en Israël, le film documentaire The Ancestral Sin (Deri, 2017) a permis au grand public de connaitre l'histoire de la ségrégation telle qu'elle a été organisée par l'État israélien dès les années 1950 (ce dernier ayant délibérément orchestré l'installation des immigrés juifs originaires d'Afrique du Nord et du MoyenOrient dans des villes périphériques comme Kiryat Shmona) : une histoire largement méconnue jusqu'à récemment.

12 Kiryat Shmona a été fondée en 1949 sur les ruines du village palestinien Al Khalisa, et ce pour accueillir les immigrés juifs maghrébins et moyen-orientaux qui arrivent alors en Israël (Abbasi, 2016; Goldstein, 2015). Souffrant de la distance avec le centre du pays $^{12}$ (qui pourvoit une majorité des emplois) et située en première ligne des conflits 
et guerres israélo-libanais, la ville subit un exode continu compensé régulièrement par l'installation d'une nouvelle vague d'immigrés. La dernière en date est l'immigration post-soviétique. Encore aujourd'hui, $16 \%$ des habitants de la ville sont arrivés d'exURSS après 1989 (CBS, 2016).

Le poids démographique des habitants d'ex-URSS leur a permis une représentation politique continue au sein du conseil municipal. Ces dernières années, c'est le Dr. B, adjoint au maire de la ville, ainsi qu'une conseillère municipale elle-même immigrée, qui représentent cette communauté russophone. Tous deux sont affiliés au parti Israel Beitenu, un parti nationaliste russophone, dont la ligne politique consiste en la défense des droits socio-culturels des immigrés russophones d'une part, et une approche sévère "Hawkish ${ }^{13}$ en terme de politique étrangère d'autre part ${ }^{14}$.

Dans ce contexte, la réalisation de ce film a permis de répondre à des enjeux épistémologiques abordés tout au long de la recherche doctorale. Le premier enjeu concerne la mise en visibilité des acteurs et des interactions sociales, dans des lieux marginaux. Le film s'inscrit dans une démarche qualitative complémentaire à des entretiens et des observations, afin de donner à entendre les acteurs locaux, et de mieux discerner leur rôle dans l'élaboration des politiques locales d'accueil des immigrés. Le deuxième enjeu correspond à l'importance de la position structurelle de Kiryat Shmona en périphérie et à la frontière du territoire israélien. La production d'images en mouvement dans des lieux éloignés de la capitale économique Tel Aviv, ou de la ville disputée de Jérusalem, permet de mieux rendre compte de la vie sociopolitique d'une ville périphérique. Enfin, le film a offert la possibilité d'une lecture «à la marge » de problèmes qui touchent la région, tels que l'immigration, et même plus largement le conflit israélo-palestinien.

\section{Filmer caméra au poing : le mouvement révélateur d'inertie?}

Dans ce contexte épistémologique j'ai choisi de privilégier une approche narrative. En effet, le film se déroule dans une seule ville, Kiryat Shmona, et a pour personnage principal un seul élu local, l'adjoint au maire de la ville, lui-même immigré. En acceptant d'être filmé, cet homme se « met en scène » et organise en partie un récit - en partie, puisque nous sommes tous deux responsables des images produites.

De fait, une question centrale de l'ethnographie visuelle est celle de la relation de tournage entre filmant et filmé, symbolisée matériellement par la caméra. Dès les années 1960 , le travail de Jean Rouch opère un renversement méthodologique crucial en ce domaine.

17 Jean Rouch, ethnologue cinéaste français (1917-2004) a imaginé le terme de ciné transe qui a fait fureur depuis, pour décrire la fusion entre le corps du chercheur et sa caméra lorsqu'il filme ses interlocuteurs, en mouvement :

« (...) pour moi, donc, la seule manière de filmer est de marcher avec la caméra ; de la conduire là où elle est le plus efficace, et d'improviser pour elle un autre type de ballet où la caméra devient aussi vivante que les hommes qu'elle filme. (...) Le caméraman réalisateur pénètre réellement dans son sujet, précède ou suit le danseur, le prêtre ou l'artisan, il n'est plus lui-même mais un "œil mécanique" accompagné d'une "oreille électronique". C'est cet état bizarre de transformation 
de la personne du cinéaste que j'ai appelé, par analogie avec les phénomènes de possession, la "ciné-transe" » (Rouch, op.cit. : p. 63) (de Hasque, 2014, p. 43). questions. Dans des travaux plus récents, Jean-Frédéric de Hasque compare la relation filmant-filmé à une danse (2014), alors que Fabienne Le Houérou, elle, parle d'une «créativité partagée » $(2012$, p. 8$)$ où les acteurs décident de la mise en scène. De Hasque définit la démarche filmée au travers d'une posture impliquée et participante (Hasque et al., 2014, p. 42). Le corps du filmant, qui porte la caméra, est sollicité autant que celui du filmé. S'établit alors une connivence avec le filmé, qui fait comme s'il ne voyait pas la caméra, décrit De Hasque. En effet, le Dr. B. regarde rarement dans la direction de la caméra, sans jamais en ignorer complétement la présence. Réciproquement, De Hasque montre que le filmeur devient lui-même partie intégrante en «dansant » en même temps que le danseur, ou plus généralement, en suivant les mouvements des personnages du film pour les saisir (ibid.). Par exemple, en suivant le Dr. B. de bureau de vote en bureau de vote, j'accélère le pas quand il accélère le pas, je me tourne vers la direction dans laquelle il regarde, je m'insère dans les cercles qui se créent autour de lui.

Le Houérou décrit un projet commun, collectif. Cependant, pour elle, cette créativité partagée est liée à une parole libérée, sans « direction » du chercheur, qui permet aux filmés de produire une parole neuve. Cette démarche méthodologique répond à la nécessité de restaurer pour ses interlocuteurs une forme de contrôle et d'estime de soi, alors même que ces derniers sont victimes de migrations forcées, et que la chercheuse évolue sur des terrains dits sensibles. Néanmoins, cette première expérience caméra au poing a en partie réfuté cette notion de "parole libérée ». Au contraire de ce que Le Houérou décrit, je me suis rendue compte qu'en filmant, je chorégraphiais. En effet, le filmé est supposément libre de se déplacer et d'emmener celui qui le suit caméra au poing avec lui. Mais dans le cadre du tournage avec le Dr B., ses déplacements, ses regards pour s'assurer que je suis bien là, que j'ai bien entendu, semblent aussi des réponses à ce que le filmé croit que j'attends de lui. En effet, la présence de la caméra change en partie l'attitude du Dr. B., si je la compare à nos interactions à la mairie qui ont eu lieu à de multiples reprises avant le tournage du film, sans caméra, seulement avec un dispositif audio. En effet, il se met plus en scène et il s'autorise certains discours qui n'avaient pas leur place lors des entretiens (voir section suivante). De plus, lors du visionnage du film (voir la dernière section de l'article), le Dr. B. semble se préoccuper de son apparence. Même sans diriger de façon explicite le film, l'accès à une parole «débridée » qui serait permise par la caméra (Le Houérou, 2012) peut au contraire être difficile.

Avant de décrire le tournage, il semble important de préciser que j'avais déjà rencontré le Dr. B. à plusieurs reprises dans son bureau, d'abord pour un premier entretien dont l'objectif était de recueillir un récit de vie. Nous avons ensuite effectué une seconde rencontre sous forme de déambulation dans la ville. Ensuite, à chacune de mes visites à Kiryat Shmona, je prenais le temps de venir le saluer et de discuter des dernières nouvelles de la ville. Ce n'est qu'à la suite de ma première formation sur le film ethnographique que je l'ai sollicité et lui ai demandé s'il acceptait d'être l'objet d'un documentaire. Un élément important qui a sans doute orienté sa réponse, au-delà de notre familiarité, est son identité de personnage public. Au moment de mon appel, il venait tout juste d'être filmé pour un reportage produit par une chaîne nationale israélienne. En tant qu'élu local, il est aussi photographié et filmé régulièrement lors de 
visites officielles ou d'évènements publics liés à l'avenir de Kiryat Shmona. Le Dr. B. savait donc de quoi il retournait lorsque je lui proposais ce tournage, mais il était aussi flatté d'être au centre de l'attention.

21 Après avoir obtenu son autorisation, je retrouve le Dr. B. le jour des élections nationales, élections anticipées qui ont suivi la dissolution du gouvernement dont certains membres refusaient le débat sur la loi de l'État-nation ${ }^{15}$. Il est très tôt, et aucune banderole, aucune tente n'est encore installée. J'accroche un micro au col du Dr. B. et nous nous rendons vers les différents bureaux de vote. Dr. B. et des militants du parti Israel Beitenu se mettent à accrocher posters et banderoles. Les électeurs indécis doivent encore être convaincus, et ceux qui ne sont pas encore allés voter se voient rappeler leur devoir de citoyens.

Avec cette caméra et ce micro-cravate, j'ai pu me rapprocher, de façon plus « intime », du quotidien d'un homme politique immigré. En effet, comme le souligne Jean-Frédéric de Hasque (2014, p. 39),

« cette démarche [l'ethnographie filmique] peut s'avérer utile et nécessaire pour récolter des données sur le terrain, par exemple, en présence d'hommes politiques pour enregistrer les creux du discours (Abélès 1989), lorsque [ces hommes politiques] doi[vent] sortir de la parole convenue émanant du pouvoir (Banegas \& Warnier, 2001), ou encore, si [le chercheur] désire saisir ce qui est de l'ordre du non-exprimé par une parole (Vuillemenot, 2011)».

En effet, la présence sur le temps long - de huit heures du matin à dix heures le soir lors du premier tournage, puis durant deux demi-journées lors d'un deuxième tournage - permet de saisir des moments creux: la préparation de la journée au bureau, l'accrochage des banderoles et le montage de la tente, le déjeuner, les temps en voiture d'une urne à l'autre... Autant d'images enregistrées alors même que le Dr. B. ne pense pas être en situation de représentation.

Suivre le Dr. B. lors de cette journée m'a permis d'enregistrer les dialogues avec les résidents, loin du face à face de son bureau. Lors de la journée d'élection, chaque résident russophone connu du groupe de militants est abordé, pour lui rappeler de voter pour le parti, et d'introduire le L (Lamed en hébreu) le symbolisant dans l'urne ${ }^{16}$. C'est l'occasion de demander des nouvelles de la famille, mais aussi pour les votants, de demander des explications en russe: les personnes âgées en particulier qui ne maîtrisent pas bien l'hébreu étaient un peu désorientées et demandaient leur chemin, à quel bureau de vote elles appartenaient et d'autres détails pratiques. Ces conversations banales, qui me seront traduites plus tard en visionnant les enregistrements, n'auraient pas pu être transcrites immédiatement dans mon carnet de terrain. Bien que l'enregistrement audio le permette, l'avantage comparatif de la caméra est de montrer la façon dont ces interactions se forment, le degré de familiarité des interlocuteurs, et de repérer, grâce à l'image, les superpositions de scènes et de dialogues.

Lors de cette journée, les différentes fonctions du Dr. B. s'alternent. D'abord, il est le représentant du parti dans la ville et la région alentour - ce pourquoi il a organisé la campagne avec d'autres membres actifs. Il navigue d'un bureau à l'autre pour s'assurer que tout le monde s'affaire. Mais il est aussi un conseiller municipal aux aguets, vérifiant la bonne tenue du vote, la présence d'un garde à l'entrée, ou encore l'absence de banderoles au-delà de la grille du bureau de vote. Au gré des rencontres avec d'autres partisans, les railleries s'enchaînent, et ceux qui travaillent d'habitude ensemble à la mairie se trouvent soudain en compétition. La présence de la caméra pousse à la surenchère : les groupes me prennent à témoin pendant leur joute verbale, 
et personne n'a l'air de s'inquiéter alors du politiquement correct. Les différents militants usent de l'origine nationale ou de l'appartenance religieuse, et rient des stéréotypes affublés les uns aux autres, en me jetant un œil pour s'assurer que leur boutade a bien été enregistrée. Enfin, en tant qu'ancien médecin urgentiste et résident du quartier des «Roses », un quartier construit lors de l'arrivée des juifs ex-soviétiques, le Dr. B. s'enquiert en bon voisin de la santé de chacun.

Un moment important du tournage a été la visite de la députée Orly Levy-Abukassis, numéro 2 du parti Israel Beitenu. Cette députée est la fille du parlementaire d'origine marocaine David Levy et elle-même résidente de Galilée (ville de Beit Shean). La présence de Levy-Abukassis dans un parti russophone est symbolique du virage pris récemment par le parti Israel Beitenu. Alors que son électorat russophone est vieillissant (Gleizer, 2015) - et le film rend bien compte de cette tendance démographique -, le parti a adopté une stratégie politique en direction des marges, c'est-à-dire des populations originaires des pays du Maghreb et du Moyen-Orient, et des résidents des villes périphériques. L'implantation solide d'élus locaux comme le Dr. B. lui permet de récolter des voix. En effet, et les conversations enregistrées par le micro-cravate du Dr. B. avec Levy-Abukassis reflètent bien cette réalité, l'électorat soutient Israel Beitenu plus pour soutenir le Dr. B., que parce qu'il tient sincèrement à la politique ultranationaliste menée par le leader du parti, le parlementaire Avigdor Lieberman. Grâce au micro-cravate, j'ai pu entendre la description que le Dr. B. faisait à Levy-Abukassis d'une campagne éminemment locale, qui le place en concurrence avec le maire, affilié Likud $^{17}$. Lors de sa visite, les résidents apostrophaient Levy-Abukassis. Certains ne comprenaient pas son choix de rejoindre un parti russophone alors que celle-ci ne maitrise pas le russe, source de frustration pour l'électorat traditionnel. Là encore, le clivage Marocains-Russes, reflet du clivage national Mizrahi-Ashkénazes ${ }^{18}$, était au cœur des conversations à la sortie des urnes.

Lors de la visite de Levy-Abukassis, le Dr. B. se trouve dans une relation de pouvoir différente, puisqu'un membre plus élevé que lui dans la hiérarchie du parti vient visiter la ville. A ce moment-là, le Dr. B. a oublié la caméra, et j'ai alors dû me faufiler à travers la foule des administrés pour le rattraper. Son attention s'est tournée vers la visiteuse, à laquelle il souhaitait montrer ses efforts pour que les résidents de Kiryat Shmona soutiennent le parti Israel Beitenu. Il a continué de «jouer », non plus pour le film mais pour sa supérieure. Fait intéressant, ni elle, ni son chauffeur, ne m'ont posé de questions ni ne m'ont demandé ce qu'il allait advenir des enregistrements. J'essayais de rester dans la portée du signal du micro-cravate, et de continuer à filmer alors que les résidents présents s'approchaient et cherchaient à capter l'attention de LevyAbukassis.

27 À la fin de la journée, lors du dépouillement, les résultats se font attendre. Le petit groupe était fatigué, mais a quand même organisé un pique-nique au bureau local, installé dans une ancienne salle de restaurant. Derrière la caméra, je me suis rendue compte de l'impatience grandissante du Dr B. Lorsque les résultats sont enfin arrivés, ce dernier s'est étonné de voir que je filmais toujours: il m'avait oubliée. Ainsi, le réalisateur belge Jean Libon (auteur du film Ni juge, ni soumise (2018) centré sur la juge Anne Gruwez) alors interviewé par France Culture, explique pourquoi le personnage principal ne regarde plus la caméra: "parce que ce qu'elle [la juge Gruwez] vit à ce moment-là, est plus important que nous " (Gesbert, 2018). En effet, face aux enjeux des élections, on voit alors le Dr. B. plus fatigué, et aussi déçu. Il tente vainement 
d'actualiser la page internet affichée sur son ordinateur pour y lire les derniers résultats. Tous les militants espéraient que les élections infirmeraient les intentions de vote, qui ne cessent de diminuer pour ce parti. Néanmoins, Israel Beitenu obtient ce jour-là 7 sièges sur les 120 de la Knesset.

Lorsque je reviens avec la caméra deux mois plus tard, à l'occasion des commémorations de la Seconde Guerre mondiale, les raisons de cette perte de vitesse se font plus évidentes. La communauté russophone se réduit et est vieillissante. Les personnes âgées sont celles qui restent à Kiryat Shmona. Les jeunes sont partis vers le centre, et comme le Dr. B. l'avait souligné lors d'un précédent entretien, Kiryat Shmona devient une grande maison de retraite.

Lors de la cérémonie, le Dr. B. est entouré du maire de la ville, du second adjoint au maire, et d'anciens combattants de l'ex-URSS. Dans son discours de commémoration, prononcé en hébreu, le maire de la ville, né au Maroc et arrivé très jeune en Israël, adopte un ton bien différent de celui qui prévalait lors des élections. Il présente les anciens combattants de l'armée rouge comme des combattants transnationaux. Il loue leur participation à l'établissement d'Israël: ces derniers ont libéré l'Europe du nazisme, assène-t-il, et sans eux, la Shoah aurait fait encore plus de victimes. Le déjeuner organisé à la mairie après la cérémonie rend ce rapprochement entre anciens combattants de l'armée rouge et résidents de Kiryat Shmona absurde: le maire et quelques conseillers présents ne comprennent rien aux discours et histoires que les anciens combattants racontent en russe. Pour leur faciliter la compréhension, le Dr. B. les traduits ici et là, mais seulement partiellement.

Le lendemain, un petit groupe d'anciens combattants se rend dans un des lycées de la ville pour une projection d'un documentaire historique sur la Seconde Guerre mondiale, suivie de témoignages. Là encore, les témoins parlent russe, et leurs propos sont traduits par le Dr. B. ou par un responsable associatif local bilingue. Les lycéens restent silencieux, et sont manifestement peu enclins à poser des questions ou à réagir.

31 Ces deux tournages, réalisés dans des contextes bien différents, m'ont donc permis d'enregistrer de nombreuses scènes, où la caméra agit comme une "autorité », me permettant de justifier ma présence.

\section{Le glissement parodique, si l'on « n'aime pas ses indigènes " (Avanza, 2008)}

Si la production d'images en Israël pose de nombreuses questions, la production d'images autour d'un membre du parti Israel Beitenu en pose encore d'autres. Ce parti a adopté une ligne dure dans le contexte du conflit israélo-palestinien. Il s'aligne avec la politique "Hawkish» du gouvernement (Acosta, 2014; Ram, 2000; Shafir et Peled, 2002) face à la Palestine, à son idéologie nationaliste sioniste. Le slogan de la campagne de 2015, "peine capitale aux terroristes ", donne ainsi une idée de la teneur de son idéologie. C'est d'ailleurs ces grandes banderoles que le Dr. B. et ses compagnons ont accrochées à l'extérieur des bureaux de vote le jour des élections. Dès cette première heure de tournage, la question de savoir quoi filmer s'est posée. Comme l'indique Rouch, c'est l'aspect du terrain qui est unique à l'ethnographie filmique : au lieu de prendre des notes et de les faire évoluer à son retour du terrain, celui qui filme doit, s'il ne veut pas échouer, effectuer un travail de synthèse au moment même de 
l'observation (Rouch, 1974, p.41). S'il est facile de capter des images qui rendent compte de l'idéologie radicale du parti, je dois prendre du recul pour capter le quotidien du Dr. B., même un jour comme celui des élections, et ne pas adopter une attitude qui montrerait trop vite ce que je désapprouve, m'exposant au risque de me voir demander par mes interlocuteurs de baisser la caméra. Capter ces moments moins politisés est crucial pour qu'au montage, je puisse échapper à la parodie, à l'édition d'images certes réelles, mais trop stéréotypées.

L'hostilité des membres du parti Israel Beitenu envers les Palestiniens est de notoriété publique, et le Dr. B. ne s'en est pas caché. Alors que des jeunes membres du Likud lui demandaient ce que Poutine pensait du conflit, le Dr. B. leur a ainsi assuré qu'avec les armes que le président russe possède, il pourrait «détruire une partie des villages arabes ici, et on n'en demande pas plus ", avant de se tourner vers la caméra, mi-amusé mi-gêné de sa remarque, sans pour autant me demander de baisser l'objectif.

Dans ce cas précis, être derrière la caméra m'a permis de rester de marbre : la camera permet de dissimuler en partie nos réactions et nos jugements.

« Ainsi, l'emploi d'un appareillage comme une caméra, par son rôle d'intermédiaire, permet à la fois de faciliter notre insertion et notre mise en tension (en résonance) mais maintient également une distance » (de Hasque, 2014, p. 46).

S'il avait vu mon visage, le Dr. B. se serait-il censuré ? C'est probable, puisque ce type de remarques n'avait pas sa place dans nos échanges au bureau, dans le contexte de l'entretien qualitatif classique. Dans ce contexte, cacher ma réprobation se serait alors avéré plus difficile. Comme l'affirme Martina Avanza, qui a enquêté auprès de militants du parti de droite radicale de la Ligue du Nord en Italie,

"le problème de la juste 'distance' est inhérent à toute enquête ethnographique

[Bensa, 1995a]. Néanmoins, cette question se pose de manière accrue quand

l'ethnologue 'part sur le terrain' sachant que les personnes avec lesquelles il va

travailler par 'observation participante' représentent politiquement tout ce qu'il

déteste » (2008, p. 41).

L'idée n'étant évidemment pas d'approuver ce type de discours, puisque je pense que la responsabilité du chercheur est également d'induire un débat, mais de laisser dire pendant la collecte de données.

Une fois les heures de tournage prêtes au montage et à l'édition, ce paradoxe - entre le désir de respecter la bienveillance dont le Dr. B. et les autres résidents ont fait preuve à mon égard pendant les jours de tournage et au-delà, et le refus de "plaider " pour le radicalisme politique que manifestent le parti Israel Beitenu et ses membres - m'a d'autant plus questionnée. Comment montrer le Dr. B. sans tomber dans la parodie ? En effet, je souhaitais monter un produit final pour lequel je voulais recevoir l'autorisation des protagonistes pour pouvoir le diffuser, tout en ne dissimulant pas les dérives de ce parti de la droite ultra-nationaliste. J'ai ainsi décidé d'intégrer les conversations relayant le racisme ordinaire, à condition d'y voir un dialogue, une réponse de la part d'autres résidents présents dans le cadre. J'ai évité les enregistrements dont la répétition tout au long du film aurait présenté le Dr. B. comme un personnage stéréotypé et risible. Ce sont ces questions de montage que je présente dans la prochaine section. 


\section{Le montage pour rendre compte de la problématique socio-culturelle}

Cinq mois ont passé lorsque je retrouve le groupe de doctorants avec lequel je participe à la formation sur le film ethnographique. Evidemment, les images sans explication ni contexte ont peu de portée : quelle ville est Kiryat Shmona, que disent les acteurs, quels sont les enjeux ? Si Israël est largement connu à travers le conflit israélo-palestinien, la vie dans une petite ville de périphérie reste une énigme pour mes interlocuteurs. Pour un public qui ne parle pas la langue, l'utilisation de l'hébreu ou du russe ou encore les accents, sont des informations inaudibles. Les pancartes sont illisibles. Pour le groupe de doctorants qui partage les heures de formation avec moi, les images les plus percutantes sont celles des deux chansons entonnées par des collégiens de la ville russophone : deux chansons patriotes connues, pour lesquelles la petite chorale chante un couplet en russe puis un en hébreu avant les discours commémoratifs. La musique et les gestes permettent de comprendre qu'il s'agit là d'une commémoration, sans qu'il soit nécessaire de traduire la scène.

Mais c'est justement la participation à une formation dans un groupe où personne ne comprend l'hébreu qui m'a obligée à réfléchir à un schéma narratif rendant compréhensible les situations, non seulement en y ajoutant des sous-titres, mais aussi en choisissant l'ordre du montage. Ainsi, démarrer par les commémorations, en ne respectant pas l'ordre chronologique, permet de situer ce groupe de résidents. On y voit alors un groupe d'anciens combattants et quelques résidents actifs dans le monde associatif de la ville, aux côtés des élus - le maire et ses deux adjoints. La chorale représente la nouvelle génération, née en Israël mais dont les parents parlent russe à la maison. Entre ces deux générations, il existe d'amples mobilités internes : une fois les études terminées, nombreux sont ces Israéliens nés de parents originaires de l'ex-URSS à partir vers le centre du pays. Preuve de leur intégration, ils désertent aussi le parti russophone pour se tourner vers des partis politiques israéliens (bien que de droite en général) (Lerner, 2015).

Dans ce contexte de vieillissement de la population et de migrations intra-nationales importantes, le Dr. B. peine à mobiliser l'électorat d'Israel Beitenu. Jusqu'au tournage du film, je ne connaissais que son rôle d'adjoint au maire, et de représentant des résidents russophones de la ville. Le film révèle un autre enjeu: sa réputation au niveau local a un impact sur les votes lors d'élections nationales. Comme il le suggère lui-même, les électeurs, lorsqu'ils mettent le bulletin d'Israel Beitenu dans l'urne, renouvèlent leur confiance en lui. En discutant avec Levy-Abukassis, l'adjoint au maire révèle aussi que les votes pour le parti chutent d'année en année.

L'arme du Dr. B., dans cette petite ville dite "de développement ", où ont surtout été installés des immigrés d'Afrique du Nord et d'Asie, c'est le communautarisme local. Le Dr. B. décrit une situation inégale, dans laquelle les "Marocains" sont comme une grande famille, et où le clientélisme obstrue les possibilités professionnelles. Il ajoute, lors d'une de nos discussions, qu'il a appris récemment que les Israéliens émigrés du Maroc sont en fait moins nombreux au niveau national que les immigrés de l'ancienne Union soviétique. La vie à Kiryat Shmona lui avait pourtant laissé penser qu'ils composaient une majorité écrasante dans le pays (sic). Il instrumentalise ainsi le clivage Mizrahi-Ashkénaze de la société israélienne afin de pouvoir s'ériger en rempart, en gardien de la communauté russophone. Pendant le tournage, les joutes entre les 
«Russes » et les "Marocains" - bien qu'évidemment, les variations géographiques soient bien plus larges (Goldberg; Bram, 2007), et que ceux nés «là-bas » ne forment plus une majorité - sont ordinaires. Par exemple, lors d'une rencontre filmée avec des représentants du parti sépharade Shas (sur le Shas, voir Zrehen, 2004), le Dr. B. se moque du fait que malgré les réseaux importants de relations des membres de Shas, notamment au Ministère de l'Intérieur, Shas n'obtient pas plus de sièges au parlement.

\section{Retour sur des images triviales} mairie de Kiryat Shmona. J'ai enregistré notre conversation à cette occasion, pour pouvoir la retranscrire. Quelques semaines plus tard, nous l'avons revu une deuxième fois dans le bureau du maire, un vendredi, alors que personne d'autre n'était présent à la mairie. Bien que je n'aie pas eu d'explication quant à cet horaire particulier, je me suis demandée si le maire ne souhaitait pas voir ce film avant qu'il ne circule. En effet, c'est l'unique occasion, pendant le travail de terrain, où j'ai été invitée à une rencontre pendant le week-end.

41 Je redoutais un peu de montrer le film, mais mon appréhension s'est vite dissipée : la première inquiétude de ceux qui se regardent à l'écran, c'est leur photogénie. Lors du premier visionnage, le Dr. B. se plaint alors de son embonpoint, et me répète qu'il doit perdre du poids. Puis il commence à me décrire plus en détails les occupations des uns et des autres. Ainsi, les militants des autres partis politiques filmés en ce jour d'élections prennent une autre dimension, où celui-ci est entraîneur de football, et celui-là professeur des écoles. Il m'explique même que certains des « militants » sont en fait payés pour faire campagne, mais votent pour un parti différent une fois devant les urnes. Ici, la pertinence du journal de terrain en parallèle au film ethnographique prend toute son ampleur: elle me permet d'en apprendre plus et de "lire entre les lignes ". qu'« on n'y apprend rien, c'est comme chaque élection». A première vue, cette remarque sur la trivialité des scènes me surprend. Néanmoins, il semble que ce soit plutôt positif d'entendre que ce que j'ai produit correspond à des images et représentations que le maire approuve. Cela dit, ce dernier se prend au jeu, et commence lui aussi à chercher les gens qu'il connaît, et à commenter les scènes. Les deux scènes où le Dr. B. s'attaque aux Palestiniens le font rire jaune, et il rappelle à l'ordre son adjoint, lui disant en souriant que ce qu'il a dit n'est pas "politiquement correct ».

En ce sens, l'idée de produire un documentaire pour pouvoir présenter un « objet » lors d'entretiens fonctionne: en jouant le jeu du décryptage des scènes, le maire et son adjoint dévoilent «leur vérité » sur certains des meneurs de campagne, discutent des résidents et des élections. Mais, étrangement, une fois le visionnage terminé, j'observe un resserrement vers un discours institutionnel. Le Dr. B., en porte à faux par rapport au maire de la ville, trouve important de raconter les expériences positives qu'il a vécues lors de son arrivée en 1993 : il parle d'activités menées par les résidents pour aider les nouveaux immigrés à s'acclimater. Le maire, rabbin dans une des communautés de la ville, se souvient lui-même d'avoir accompagné une famille, et d'avoir emmené les enfants à la synagogue le samedi. Ils se félicitent l'un l'autre de la 
capacité des résidents à se mobiliser et à accueillir les nouveaux venus, dans une perspective de vivre-ensemble. Pourtant, lors des entretiens précédents, le Dr. B. déplorait l'absence de la municipalité dans l'accueil des nouveaux immigrés ces dernières années.

L'emploi d'une méthode de collecte filmique a donc permis de mieux cerner les ambivalences entre le discours officiel et institutionnel, le discours politique et l'expérience personnelle du Dr. B. Ce dernier doit ménager les différents intérêts en jeu à Kiryat Shmona, et les tensions entre son rôle d'adjoint au maire, de représentant communautaire et de membre actif du parti sont bien plus présentes dans le matériel audio-visuel collecté que dans les entretiens classiques que j'ai pu mener avec lui. Visionner le film ensemble a ainsi permis de débattre de ces contradictions ensemble.

En revanche, il m'a été plus difficile d'obtenir des retours en dehors de la sphère israélienne. Ainsi, le documentaire a été visionné lors d'une conférence internationale organisée à Bilbao (Espagne) en juillet 2016. A cette fin, j’ai édité le film pour y faire apparaître deux bandes de texte : une première au début du film qui situe la ville de Kiryat Shmona et la commémoration, puis une seconde le jour des élections, qui explique le rôle du Dr. B. dans la campagne du parti Israel Beitenu. La salle s'est montrée plutôt crispée à la vue du cynisme et du racisme ordinaire. Il est vrai que les études sur les partis d'extrême droite xénophobes comme celle de Avanza (2008), témoignent de la difficulté d'éprouver de l'empathie pour les personnages. Même si le Dr. B. a quitté la Russie pour cause d'antisémitisme dans le monde professionnel dans lequel il évoluait (ce qui pourrait déclencher de l'empathie), le caractère ethno-nationaliste de la politique migratoire israélienne (Shafir et Peled, 2002; Yacobi et Tzfadia, 2009; Yiftachel, 2000 ; Yonah, 2005) et de la politique d'accueil rompt avec les représentations plus habituelles de la migration. En ce sens, Victory Day est resté une méthode d'observation pour mon travail de doctorat, et un objet, qui a permis de dévoiler des tensions lors d'entretiens postérieurs. Mais sa portée au-delà du doctorat semblait limitée.

Un deuxième visionnage a eu lieu à Évry en juin 2018, à l'occasion de la conférence annuelle de l'association internationale de sociologie visuelle (IVSA). Cette fois, j'ai présenté le documentaire en parallèle d'une analyse, correspondant plus ou moins à la structure de cet article. L'accueil a été beaucoup plus favorable, et l'expérience m'a montré que ce premier film nécessitait une présentation préalable du contexte et de la démarche auprès du public, avant visionnage. Ceci peut s'expliquer notamment par le choix de l'objet du film : en filmant des lieux peu ou pas familiers, et des trajectoires individuelles peu connues, $\mathrm{j}$ 'ambitionnais de rendre visible ces lieux et acteurs; mais de par l'invisibilité même de ces lieux et acteurs, je me suis confrontée au fait que le public a moins d'éléments de compréhension pour saisir la complexité des relations dans ce lieu.

\section{Conclusion}

Il est évident que le premier avantage de la caméra est d'apporter une expérience sensorielle très différente du texte - à la fois pour le chercheur, le(s) filmé(s) et pour le public. À travers Victory Day, la ville de la Kiryat Shmona et ses résidents prennent corps et deviennent visibles. Cette question de la visibilité est d'autant plus importante que Kiryat Shmona, petite ville frontalière israélienne, est quasi invisible, alors que le 
conflit israélo-palestinien est l'un des plus médiatisés en Europe et en Amérique du Nord. Retranscrire cette expérience sensorielle - le type d'architecture en ville, le paysage de la Galilée du nord, le type de commerces ou d'infrastructures, les musiques qui émanent des magasins, et les accents et langues entendus dans la rue - est permis par la caméra, qui enregistre et autorise l'agencement des images via le montage.

Au-delà du seul fait de montrer Kiryat Shmona, Victory Day met aussi en scène un élu local, immigré de l'ancienne URSS. L'immigration post-soviétique, si elle a eu un retentissement extrêmement important auprès des chercheurs en sciences sociales, et en particulier, auprès de ceux qui s'intéressent aux migrations internationales, n'est pas forcément visible dans l'espace médiatique, que ce soit en Israël (Gershenson; Hudson, 2008) ou dans le monde. Pour le Dr. B., la participation à un petit film amateur est une source de fierté (Le Houérou, 2012). D'ailleurs, dans un contexte d'abandon d'une politique d'accueil à Kiryat Shmona, ma présence et les entretiens que j'ai conduits participent à consolider ses revendications pour une politique locale d'accueil plus explicite, et mieux financée (Desille, 2017).

L'utilisation de la caméra a aussi montré ses limites pour rendre compte de la complexité et de la stratification socio-culturelle et politique israélienne. Sans aucun "capital» préalable, je présume qu'il est difficile de se rendre compte de cette complexité. Le film propose des mises en scènes, mais le risque d'adopter un style parodique ou stéréotypé me semblait élevé. L'idée n'étant évidemment pas de reproduire les fractures Askénaze/Mizrahi, israélien/nouvel immigré, laïc/religieux ${ }^{19}$ à travers le scénario, mais plutôt d'en expliquer la portée dans le contexte social, culturel et politique d'une ville comme Kiryat Shmona. Là, la nécessité d'avoir plusieurs méthodes de collecte, d'analyse et de restitution s'est imposée.

En revanche, dans ce contexte particulier, où il est difficile d'observer sans prendre part, la caméra devient un atout. La distance politique entre les chercheurs et les partis radicaux qu'ils peuvent examiner se matérialise à travers la caméra, objet qui permet le rapprochement, mais qui maintient la distance. À ceci s'ajoute la possibilité, comme l'ont souligné plusieurs chercheurs, de

« récolter des données sur le terrain, par exemple, en présence d'hommes politiques pour enregistrer les creux du discours (Abélès, 1989), lorsque l'on doit sortir de la parole convenue émanant du pouvoir (Banegas; Warnier, 2001), ou encore, si l'on désire saisir ce qui est de l'ordre du non-exprimé par une parole (Vuillemenot, 2011)» (De Hasque, 2014 : p. 39).

51 Enfin, lorsqu'il s'agit de partager les résultats de sa recherche, et ce dans une démarche participative, le film fournit une méthode intéressante. En visionnant le film avec le maire et son adjoint, j'ai pu recueillir leurs réactions, mais aussi favoriser un dialogue sur les questions que le film soulève, telles que la représentation des immigrés ou les relations intergroupes. Mais là encore, le fait d'avoir un corpus en amont, constitué d'entretiens, d'observations et d'actualités municipales, permettait de comparer ces différents discours et de « lire entre les lignes ».

Plus largement, cette expérience de tournage a confirmé toute la pertinence et tout l'intérêt de l'introduction des outils audiovisuels en général, et du film ethnographique en particulier comme outils de collecte et de restitution des données. Néanmoins, cet outil de travail ne peut se passer ni d'une phase réflexive durant laquelle le chercheur doit interroger sa posture éthique, ni d'une phase de confrontation avec d'autres matériaux récoltés, qui permet au chercheur de ne pas considérer le film comme un 
miroir de la réalité. Au contraire, le tournage du film puis son montage révèle de nouvelles formes de relations entre chercheur et participants, entre filmant et filmés, et entre les participants eux-mêmes. Ce qui se joue entre les acteurs est encore différent des enjeux rencontrés pendant les entretiens et les observations.

\section{BIBLIOGRAPHIE}

Abbasi, Mustafa (2016) Khalsa: The Town with a 'Golden Fountain': The Rise and Fall of an Arab Town North of the Huleh Valley, The Journal of Imperial and Commonwealth History, vol. 44, $\mathrm{n}^{\circ} \mathrm{3}, \mathrm{pp}$. 448-469.

Acosta, Benjamin (2014) The dynamics of Israel's democratic tribalism, Middle East Journal, vol. 68, $\mathrm{n}^{\circ} 2$, pp. 268-286.

Avanza, Martina (2008) Comment faire de l'ethnographie quand on n'aime pas « ses indigènes »? Une enquête au sein d'un mouvement xénophobe, in Bensa, A. ; Fassin, D. (dir.) Les politiques de l'enquête, Paris, La Découverte, pp. 41-58

Berthomière, William (2001) Aliya from the Former Soviet Union, Bulletin Du Centre de Recherche Français à Jérusalem, n 8, pp. 188-218.

Berthomière, William (2004) Sionisme et immigration en Israël, Mouvements, vol. 33-34, $\mathrm{n}^{\circ}$ 3, pp. 30-35.

Central Bureau of Statistics (2016) Statistical Abstract of Israel 2016, Jerusalem, Central Bureau of Statistics [Disponible sur Internet].

Chetrit, Sami Shalom (2000) Mizrahi Politics in Israel: Between Integration and Alternative, Journal of Palestine Studies, vol. 29, $\mathrm{n}^{\circ}$ 4, pp. 51-65.

Deri, David (2017) the Ancestral Sin, Israel, 109 minutes [film documentaire].

Desille, Amandine (2017) Governing or being governed? A scalar approach of the transformations of State power and authority through the case of immigration and integration policies of four frontier towns in Israel, Poitiers, Université de Poitiers, 429 p., Th. Doct: Géogr.: Poitiers: 2017.

Desille, Amandine (2019) Immigration and the city: between state planning and residents' practices and representations: The case of Kiryat Shmona in Israel, GeoJournal, vol. 84, $\mathrm{n}^{\circ} \mathrm{2}$, pp. 437-457.

Gehin, Jean-Paul ; Giglio-Jacquemont, Armelle (2012) Filmer le travail : chercher, montrer, démontrer, Ethnographiques, $\mathrm{n}^{\circ} 25$ [Disponible sur Internet]

Gershenson, Olga; Hudson, Dale (2008) New Immigrant, Old Story: Framing Russians on the Israeli Screen, Journal of Film and Video, vol. 60, n³-4, pp. 25-41.

Gesbert, Olivia (2018) La justice à nu par Jean Libon, La grande table, Paris, France Culture (émission du 2 janvier) [Disponible sur Internet].

Gleizer, Hilo (2015) Lieberman, niet!, Haaretz, Jerusalem (30 janvier). 
Goldberg, Harvey; Bram, Chen (2007) Sephardic/Mizrahi/Arab-Jews: Reflections on Critical Sociology and the Study of Middle Eastern Jewries within the Context of Israeli Society, in Medding, P. Y. (dir.) Sephardic Jewry and Mizrahi Jews, Oxford, Oxford University Press, pp. 227-258. Goldstein, Amir (2015), Eliezer Krol of "Ha-Shomer" and the Yemenite Immigrants in Kiryat Shmonah, 1949-1950 (in Hebrew), Iyunim Bitkumat Israel (studies in Israeli and modern Jewish society), $\mathrm{n}^{\circ} 25$, pp. 151-178.

Hasque, Jean-Frédéric de (2014) Corps filmant, corps dansant, Parcours anthropologiques, $\mathrm{n}^{\circ}$ 9, pp. 39-51.

Le Houérou, Fabienne (2012) Filmer le désarroi : ce que révèle l'usage de la caméra dans les situations « sensibles », e-Migrinter, $\mathrm{n}^{\circ} 9$ [Disponible sur Internet]

Lerner, Julia (2015) « Russe » dans l'État juif. Le sang, l'identité et l'administration nationale, Ethnologie française, vol. $45, n^{\circ} 2$, p. 363.

Pereira, Sónia; Maiztegui-Oñate, Concha; Mata-Codesal, Diana (2016) "Transformative looks": Practicing Citizenship Through Photography, Journal of Social Science Education, vol. 15, $\mathrm{n}^{\circ} \mathrm{3}, \mathrm{pp}$. 14-21.

Ram, Uri (2000) National, Ethnic or Civic? Contesting Paradigms of Memory, Identity and Culture in Israel, Studies in Philosophy and Education, vol. 19, n 5-6, pp. 405-422.

Raulin, Anne ; Conord, Sylvaine ; Berthomière, William ; Ebilitigué, Ines ; Färber, Alexa ; Ma Mung, Guillaume ; Veiga Gomes, Hélène (2016) Migrations et métropoles : visées photographiques, Revue européenne des migrations internationales, vol. 32, n 3-4, pp. 69-130.

Shafir, Gershon; Peled, Yoav (2002) Being Israeli: The Dynamics of Multiple Citizenship, New York, Cambridge University Press, $406 \mathrm{p}$.

Simon, Gildas ; Berthomiere, William ; Anteby, Lisa ; Rosenbaum-Tamari, Yehudit ; Damian, Natalia (1996) Identité juive et immigration en Israël : Une enquête en cours sur l'actuelle vague d'immigrants russes, Revue européenne de migrations internationales, vol. 12, n 3, pp. 123-138.

Weber, Sanne (2018) Participatory Visual Research with Displaced Persons: 'Listening' to Postconflict Experiences through the Visual, Journal of Refugee Studies. [Disponible sur Internet].

Yacobi, Haim; Tzfadia, Erez (2009) Multiculturalism, Nationalism, and the Politics of the Israeli City, International Journal of Middle East Studies, vol. 41, nº 2, p. 289.

Yiftachel, Oren (2000) « Ethnocracy » and Its Discontents: Minorities, Protests, and the Israeli Polity, Critical Inquiry, vol. 26, n 4, pp. 725-756.

Yiftachel, Oren; Tzfadia, Erez (2004) Between periphery and 'third space': identity of Mizrahim in Israel's development towns, in Kemp, R.; Newman \& Yiftachel (dir.) Israelis in Conflict: Hegemonies, Identities and Challenges, Brighton, Sussex Academic Press, pp. 203-235.

Yonah, Yossi (2005) Israel as a multicultural democracy : Challenges and obstacles, Israel Affairs, vol. $11, n^{\circ} 1$, pp. 95-116.

Zrehen, Richard (2004) Le Shas : une révolution culturelle et politique, Outre-Terre, vol. 9, $\mathrm{n}^{\circ} 4$, pp. 241-261. 


\section{NOTES}

1. Des formations en sociologie filmique sont ainsi disponibles, comme par exemple à l'Université Evry Val d'Essonne ou en anthropologie visuelle à l'EHESS. Une liste indicative a été recensée par Sophie Accolas pour le Journal des Anthropologues, et est disponible en ligne: https:// journals.openedition.org/jda/5603.

2. Le laboratoire Migrinter, qui accueille la revue e-Migrinter, est fortement impliqué dans ces productions. Par exemple, le colloque «Images du travail, travail des images » qui s'est déroulé à l'Université de Poitiers en novembre 2009, est suivi d'un numéro spécial Filmer le travail : chercher, montrer, démontrer (2012). Le festival «Filmer le Travail» fait aujourd'hui l'objet d'une édition annuelle à Poitiers. Plusieurs chercheurs affiliés à Migrinter ont également présenté des travaux visuels dans le dossier spécial de la REMI, intitulé 30ème anniversaire. Renouveler la question migratoire (2016) faisant suite au colloque des 30 ans organisé à Migrinter. Enfin, du 7 au 9 novembre 2018, Migrinter a organisé le colloque international « Documenter les "expériences du choc" de la migration ».

3. La Marie Curie Networks Film School, un atelier dédié au film documentaire pour la recherche sociale, s'est tenue du 1 au 5 décembre 2014 puis du 18 au 23 mai 2015 à Central European University à Budapest en Hongrie.

4. La désintégration de l'Union soviétique en 1989 a entraîné une des plus grandes vagues migratoires qu'Israël ait connue: l'arrivée, en l'espace d'une décennie, de 850000 juifs de l'ancienne URSS. Par le biais d'une politique de logement dirigiste, l'administration israélienne a encouragé l'installation de ces nouveaux immigrés vers les périphéries. La ville de l'enquête comprend, encore aujourd'hui, 16 \% d'immigrés de l'ancienne URSS arrivés dans les années 1990.

5. En 1995, le dissident politique russe Natan Sharansky, immigré en Israël dans les années 1970, fonde le parti russophone de droite Israel beAliyah (un jeu de mot qui signifie "Israël monte », ou «Israël en immigration »). Lors des élections de 1996, la moitié des électeurs de l'ancienne Union soviétique vote pour ce parti, donnant 7 sièges à la Knesset. A la suite de conflits politiques, Israel beAliyah est dissous, et laisse la place à Israel Beitenu (« Israël notre maison »), un parti fondé par l'immigré moldave Avigdor Lieberman en 1999. Il représente les immigrés russophones en Israël, laïcs, nationalistes et sionistes, et adopte une posture "va-t'en guerre » vis-à-vis du conflit israélo-palestinien.

6. Ces cérémonies sont apparues dans le paysage israélien suite à l'immigration massive de juifs de l'ancienne URSS dans les années 1990.

7. Ou, dans ce cas, le 7 mai, puisque le 9 mai 2015 était un samedi, jour de repos où la cérémonie ne peut avoir lieu.

8. Le film est disponible en ligne à cette adresse : https://vimeo.com/206026525.

9. Voir par exemple http://www.liberation.fr/evenement/1996/04/15/a-kiryat-shmona-la-peurdans-une-ville-fantome-les-habitants-se-terrent-en-attendant-la-fin-de-l-ora_168722, consulté le 14/01/2019.

10. Clip «smoke and tears " publié par le New York Times sur Kiryat Shmona pendant la guerre israélo-libanaise : https://www.nytimes.com/video/world/middleeast/1194817104940/smokeand-tears.html consulté le 14/01/2019.

11. https://www.nytimes.com/2012/08/29/sports/soccer/israels-kiryat-shmona-falls-short-ofchampions-league.html, consulté le 14/01/2019.

12. Kiryat Shmona est située à $166 \mathrm{~km}$ du district "centre». Au-delà de cette distance géographique, le Bureau Central des Statistiques en Israël classe Kiryat Shmona comme étant la 11ème ville la plus périphérique du pays, sur 252. Son indice de périphérialité (défini ici : http:// www.cbs.gov.il/hodaot2008n/24_08_160b.pdf) est qualifié de «très périphérique " (consulté le 17/06/2018). 
13. Se dit des partis politiques soutenant une politique étrangère "va-t'en guerre ", et plus particulièrement dans le cas israélien, le soutien à l'action militaire plutôt qu'à un processus de paix diplomatique avec les autorités palestiniennes.

14. Sur l'orientation politique des immigrés de l'ancienne URSS en Israël, voir par exemple Berthomière (2001), Lerner (2015), Simon, Berthomiere, Anteby, Rosenbaum-Tamari, \& Damian (1996).

15. Loi raciste qui prévoit de renforcer le caractère juif de l'État israélien, finalement adoptée en 2018.

16. En Israël, les acronymes du parti sont inscrits sur les bulletins de vote. Ainsi, le parti Israel Beitenu est représenté par la lettre hébraïque Lamed.

17. Le Likud est une formation de la droite israélienne, fondé en 1973 par Menachem Begin et Ariel Sharon. Menachem Begin devient premier ministre en 1977. Son élection marque un tournant dans la politique israélienne, puisque c'est la première fois qu'un membre d'un parti de la droite est élu. Le leader actuel du parti, Benjamin Netanyahu, est le premier ministre depuis 2009. Aux élections de 2015, décrites dans cet article, le Likud a remporté 30 sièges sur les 120 sièges de la Knesset.

18. La société israélienne est souvent décrite au prisme de ses clivages sociaux : israéliens juifs et palestiniens ; juifs ashkénazes et juifs mizrahi ; israéliens laïcs et israéliens religieux. Ces clivages se déclinent au niveau socio-économique, spatial et politique. Le clivage mizrahi-ashkénaze naît de la proto-structure sociale de l'immigration juive vers la Palestine mandataire, dès la fin du 19 ème siècle. Juifs européens ashkénazes fondent alors les premières communautés, et définissent dès lors ce qui sera le centre socioculturel du futur État. Ce clivage s'exacerbe dans les années 1950, juste après l'établissement de l'État d'Israël, alors que juifs européens ashkénazes, pour beaucoup rescapés de l'holocauste, s'installent au centre du pays, et juifs d'Afrique du nord et du Moyen-Orient sont dirigés vers des camps de transit puis des villes de développement en périphérie. Les années 1970 voient émerger un mouvement socio-politique, plus ou moins homogène, autour de cette identité « orientale " (ou mizrahi en Hébreu), qui lie immigrés juifs d'Afrique du Nord et du Moyen Orient, ségrégués spatialement, politiquement et socio économiquement. Sur ce sujet, voir Berthomière (2004), Chetrit (2000), Semyonov \& LewinEpstein (2004), Shafir \& Peled (2002) ou encore Yiftachel \& Tzfadia (2004).

19. Voir note de bas de page 18.

\section{RÉSUMÉS}

La mobilisation grandissante de l'audiovisuel en sciences sociales (Gehin; Giglio-Jacquemont, 2013) réactualise des questions qui se posent dès l'introduction de la photographie et du film ethnographique, il y a cent ans : quelle réciprocité instaurer avec les sujets filmés? Quelle est la part de l'interprétation de l'ethnographe? «Comment rendre visible sans stigmatiser?» (Raulin et al., 2016). À partir de ces questionnements, l'objectif de cet article est de proposer des pistes de réflexions sur la production d'images en mouvement, le tournage, le montage et la diffusion, notamment lorsque cette production implique la participation de personnes ayant fait l'expérience de la migration. Pour nourrir ces réflexions, l'auteur s'appuie sur un film ethnographique réalisé lors des élections nationales en 2015, dans une ville périphérique du nord d'Israël. 
INDEX

Index géographique : Israël

Mots-clés : ethnographie, enquête, méthodologie, film, éthique, politique d'accueil

\section{AUTEUR}

AMANDINE DESILLE

Chercheuse post-doctorante, IGOT / Universidade de Lisboa

amandine.desille@gmail.com 